\title{
Prevalência de síndroma de Burnout em médicos de família da Secção Regional Norte da Ordem dos médicos
}

Carlos Daniel Costa Reis*

\section{RESUMO}

Objetivos: Os médicos de família são os profissionais de saúde que realizam o primeiro contacto entre os utentes e o sistema de saúde, tendo uma interação contínua e mais próxima com os pacientes, a qual pode desencadear quadros de ansiedade e medo. Os sentimentos negativos potenciam a exaustão física e mental que poderá conduzir a um estado de Burnout. A conjuntura portuguesa recente apresenta fatores que podem potenciar o aumento da prevalência de síndroma de Burnout nos médicos de família. Este estudo procura reavaliar a prevalência de síndroma de Burnout nos médicos de família da Secção Regional do Norte da Ordem dos Médicos.

Tipo de estudo: Estudo transversal, observacional e descritivo com componente analítica.

Local: Portugal.

População: Médicos inscritos como médicos de família na Secção Regional do Norte da Ordem dos Médicos.

Métodos: Estudo realizado através de um questionário online constituído por dados demográficos, profissionais e o Maslach Burnout Inventory - Human Services Survey, validado para português, enviado aos médicos de família sob a forma de censo no dia 11 de novembro de 2016 e válido para submissão de respostas até ao dia 5 de dezembro de 2016.

Resultados: A prevalência de Burnout encontrada nos médicos de família foi de 17,0\% (IC95\%; 13,1-20,9) Os médicos de família apresentaram exaustão emocional alta de 66,0\% (IC95\%; 61,1-70,9), despersonalização alta de 45,7\% (IC95\%; 40,5-50,9) e baixa realização profissional de 48,2\% (IC95\%; 43,0-53,4]. A despersonalização associou-se ao sexo masculino e aos indivíduos com idades inferiores a 45 anos. A síndroma de Burnout também esteve associada a idade inferior a 45 anos e com uma duração da atividade como médico de família inferior a 20 anos.

Conclusões: A prevalência da síndroma de Burnout foi até quatro vezes maior que os valores obtidos em estudos anteriores. As associações encontradas são consistentes às verificadas em estudos anteriores, sendo a síndroma mais prevalente em médicos mais jovens e com menor experiência como médico de família.

Palavras-chave: Prevalência; Stress psicológico; Burnout profissional; Médicos de família; Portugal.

\section{INTRODUÇÃO}

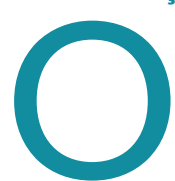

s médicos de família (MF) são profissionais de saúde qualificados que realizam o primeiro contacto dos utentes com o sistema de saúde, segundo a Definição Europeia de Medicina Familiar. São responsáveis por estabelecer a ponte entre as diversas especialidades e assistir o utente nas suas vertentes física, psicológica e social. ${ }^{1}$

*Médico Interno de Medicina Geral e Familiar. USF Sete Caminhos, ACeS Grande Porto II - Gondomar.
A interação contínua e mais próxima dos MF com o utente tem impacto sobre os profissionais de saúde. Este impacto é maior nos quadros de mau prognóstico, morte, maior exigência por parte do utente, medo, ansiedade, raiva e frustração com utentes difíceis. ${ }^{2-3}$

A persistência destas adversidades é potenciadora de exaustão e de stress físico e psicológico nos MF, que poderão conduzir a um estado de Burnout. ${ }^{4}$ A síndroma de Burnout define-se como uma resposta prolongada a fatores de stress emocionais e interpessoais no trabalho, tendo sido identificadas três dimensões: 
exaustão emocional (EE), despersonalização (DP) e realização profissional (RP). ${ }^{5}$

A ferramenta de avaliação mais utilizada para medir a síndroma de Burnout é o Maslach Burnout Inventory - Human Services Survey (MBI-HSS), um questionário constituído por questões referentes à $\mathrm{EE}$, à DP e à RP. $^{6-7}$

A existência de síndroma de Burnout pode ser definida por EE e DP altas e RP baixa. ${ }^{6,8}$

Em 2012 foi publicado um estudo que detetou uma prevalência da síndroma de Burnout, segundo a anterior definição, de 4,1\% nos MF em Portugal. ${ }^{9}$

Um outro estudo publicado em 2008, realizado em MF de 12 países europeus (não incluindo Portugal) e usando o mesmo questionário, encontrou valores de síndroma de Burnout de 12,0\%. ${ }^{10}$

A conjuntura recente de Portugal inclui alguns fatores potenciadores da síndroma de Burnout nos MF, destacando-se a crise socioeconómica em curso desde 2011, a aposentação antecipada de muitos MF com consequente contratação de novos MF e o aumento da lista de utentes destes MF mais novos.

A crise socioeconómica em Portugal teve um impacto negativo sobre a saúde dos utentes e, consequentemente, sobre a dos médicos devido ao: aumento da procura dos cuidados de saúde; incumprimento terapêutico pela incapacidade dos utentes em suportar os custos dos medicamentos; ${ }^{11-12}$ aumento da mortalidade na população idosa; aumento das taxas de suicídio; ${ }^{13-14}$ incidência de doenças infeciosas ${ }^{13} \mathrm{e}$ de patologias de distúrbios de humor e ansiedade. ${ }^{13-14}$

A distribuição etária dos médicos alterou-se com a aposentação dos médicos mais velhos, que ocupavam uma grande percentagem da população médica, e com a contratação de jovens especialistas. ${ }^{15}$ Esta alteração da distribuição, representada por uma população médica mais jovem, associada ao facto de que quanto menor for a idade do profissional maior a probabilidade para desenvolver síndroma de Burnout, pode ser um fator contributivo para o aumento da sua prevalência nos $\mathrm{MF}^{5}$

No dia 31 de dezembro de 2012 entrou em vigor o decreto-lei relativo ao aumento de número de utentes por MF: de 1.550 para 1.900 utentes ou 2.358 unidades ponderadas. No mesmo decreto-lei foi aumentada de 12 para 18 horas a parte do período normal de trabalho que pode ser dedicada às atividades urgentes e emergentes. ${ }^{16}$

Este estudo visa reavaliar o Burnout nos MF portugueses. Concretamente, os objetivos do estudo são:

1. Determinar a prevalência da síndroma de Burnout, correspondente às categorias «alta» de EE e DP e «baixa» de RP, nos especialistas em medicina geral e familiar inscritos na Secção Regional do Norte da Ordem dos Médicos (SRNOM);

2. Verificar a existência de associações entre a síndroma de Burnout, assim como das suas dimensões, e:

a) Variáveis demográficas: sexo, idade, estado civil e existência de filhos;

b) Variáveis profissionais: anos de atividade profissional, tipo de unidade em que trabalha, carga horária, tempo de contacto com utentes, número de utentes da lista e número de colegas MF na mesma unidade.

\section{MÉTODOS}

Esta investigação trata-se de um estudo transversal, em forma de censo, aos especialistas em medicina geral e familiar (MGF) inscritos na SRNOM, que decorreu entre 11 de novembro de 2016 e 5 de dezembro de 2016. A SRNOM autorizou, para a realização do estudo, a contagem dos especialistas da população e a intermediação do acesso aos mesmos por correio eletrónico.

Foi enviado no dia 11 de novembro de 2016 aos 1.976 especialistas de MGF inscritos na SRNOM um formulário online de resposta anónima, constituído por um questionário sobre dados demográficos e profissionais e o MBI-HSS, um questionário constituído por 22 questões, cotadas de 0 a 6 , das quais nove questões são referentes à EE, cinco à DP e oito à RP, validado para Portugal. ${ }^{7}$ Todos os itens do MBI-HSS eram de preenchimento obrigatório para a efetiva submissão do formulário online.

Foram excluídos, através da resposta obtida a questões iniciais do formulário enviado, os especialistas que trabalham exclusivamente em meio hospitalar, em unidades de convalescença ou no setor privado. Os médicos que se encontravam a realizar a formação específica em MGF, reformados ou sem exercer funções clínicas há mais de um mês (por motivo de doença ou por licença) foram também excluídos do estudo. 


\begin{tabular}{|c|c|c|}
\hline Variáveis (.$^{\circ}$ respostas obtidas) & & $n(\%)$ \\
\hline Sexo $(n=354)$ & Feminino & $227(64,1)$ \\
\hline \multirow[t]{4}{*}{ Estado civil $(n=356)$} & Solteiro(a) & $46(12,9)$ \\
\hline & Casado(a)/União de facto & $263(73,9)$ \\
\hline & Divorciado(a) & $42(11,8)$ \\
\hline & Viúvo(a) & $5(1,4)$ \\
\hline Idade $(n=347)$ & $>45$ & $209(60,2)$ \\
\hline Existência de filhos $(n=356)$ & Sim & $286(80,3)$ \\
\hline $\begin{array}{l}\text { Anos de atividade profissional (desde obtenção } \\
\text { de título de especialista) }(n=354)\end{array}$ & $\leq 20$ & $181(51,1)$ \\
\hline \multirow[t]{2}{*}{ Tipo de unidade em que trabalha $(n=353)$} & USF & $281(79,6)$ \\
\hline & UCSP & $72(20,4)$ \\
\hline \multirow[t]{3}{*}{ Carga horária semanal contratual total $(n=354)$} & $\leq 35$ & $92(26,0)$ \\
\hline & 36 a 40 & $150(42,4)$ \\
\hline & $\geq 41$ & $112(31,6)$ \\
\hline \multirow{3}{*}{$\begin{array}{l}\text { N. }{ }^{\circ} \text { de horas médias por dia de contacto } \\
\text { presencial com utentes }(n=357)\end{array}$} & $\leq 5$ & $6(1,7)$ \\
\hline & 6 a 8 & $290(81,2)$ \\
\hline & $\geq 9$ & $61(17,1)$ \\
\hline \multirow[t]{5}{*}{ N. ${ }^{\circ}$ de utentes da lista $(n=358)$} & $\leq 1.000$ & $3(0,8)$ \\
\hline & 1.001 a 1.500 & $13(3,6)$ \\
\hline & 1.501 a 1.750 & $95(26,5)$ \\
\hline & 1.751 a 2.000 & $225(62,8)$ \\
\hline & $\geq 2.001$ & $22(6,1)$ \\
\hline $\begin{array}{l}\text { N. }{ }^{\circ} \text { de colegas médicos de família na mesma } \\
\text { unidade }(n=358)\end{array}$ & $\leq 6$ & $211(58,9)$ \\
\hline
\end{tabular}

Legenda: USF = Unidade de Saúde Familiar; UCSP = Unidade de Cuidados de Saúde Personalizados.

Existiam especialistas de MGF da área da SRNOM que não se encontravam inscritos no Colégio de Especialidade, não sendo considerados como especialistas de MGF pela SRNOM (nem por nenhuma outra secção regional da Ordem dos Médicos). Estes especialistas não foram incluídos no estudo.

Nos dados demográficos constavam o sexo, a idade, o estado civil e a existência de filhos.

Nos dados profissionais constavam os anos de atividade profissional como MF, o tipo de unidade em que trabalha, a carga horária contratual semanal, o número médio de horas diárias de contacto com utentes e o número de colegas MF na mesma unidade. Também foi incluído o número de utentes da lista do MF. A descrição e a operacionalização das variáveis em estudo encontram-se no Quadro I.

Quanto ao questionário MBI-HSS, as pontuações obtidas nas questões correspondentes à mesma dimensão foram somadas, criando uma pontuação global de cada dimensão e posteriormente categorizada como «baixa, «intermédia» e «alta», segundo os pontos de corte usados pelo estudo europeu e estudo português. A EE foi classificada como «baixa», «intermédia» e "alta» se a pontuação global obtida foi inferior ou igual a 13 , entre 14 e 26 e maior ou igual a 27 , respetivamente. A DP foi classificada como «baixa», «intermédia» e «alta» se a pontuação global obtida foi inferior ou igual a 5, entre 6 e 9 e maior ou igual a 10 , respetivamente.ARP foi classificada como «alta», «intermédia»e «baixa»se a pontuação global obtida foi inferior ou igual a 33, entre 34 e 39 e maior ou igual a 40 , respetivamente. ${ }^{9-10}$ Posteriormente, as dimensões EE e DP foram recodificadas em variáveis categóricas, «alta»e «não alta», e RP em «baixa» e «não baixa» para análise estatística.

Para maximizar a taxa de resposta foram enviados dois lembretes para preenchimento do formulário a todos os especialistas, independentemente da resposta prévia. Para manter o anonimato e simultaneamente evitar respostas em duplicado, os lembretes foram emitidos num curto espaço de tempo, ao $10^{\circ}$ e ao $18^{\circ}$ dia depois do primeiro envio (dias 21 e 29 de novembro de 2016), pedindo explicitamente que não voltasse a res- 
ponder quem já tinha respondido.

Os dados recolhidos foram exportados para um ficheiro informático de Microsoft Office Excelß 2010 e convertidos para o IBM® Statistical Package for the Social Sciences (SPSS) ${ }^{\circledR}$ Statistics v. 23, 2015, onde foi realizada a análise estatística.

As variáveis idade, anos de atividade profissional e número de colegas MF na mesma unidade funcional, recolhidas como contínuas, foram convertidas em variáveis categóricas para melhor comparação com outros estudos. ${ }^{9}$ Nas variáveis categóricas ordinais, as categorias com $n$ baixo foram agregadas às categorias mais próximas com $n$ aceitável. A variável categórica do estado civil foi transformada em variável dicotómica com as categorias «não casado/união de facto» $\mathrm{e}$ «casado/união de facto».

Foi usada a estatística descritiva para descrever as características dos especialistas de MGF através de tabelas de frequência. Foi calculada a frequência dos vários graus (alto, intermédio e baixo) de cada dimensão, assim como a prevalência da síndroma de Burnout. Os intervalos de confiança a $95 \%$ para a frequência de cada dimensão e síndroma de Burnout foram calculados através do T test.

Para explorar a associação das variáveis com a EE alta, DP alta, RP baixa e síndroma de Burnout foi utilizado o teste qui-quadrado de Pearson para as variáveis dicotómicas e tendência linear qui-quadrado para as ordinais, com significância estatística para valores de $p$ inferior ou igual a 0,05 .

$\mathrm{O}$ anonimato dos especialistas participantes foi garantido através da intermediação da SRNOM que enviou o mailing mas não participou no tratamento das respostas recebidas. O consentimento informado foi garantido através da aposição de informações sobre o estudo no cabeçalho do questionário e da devolução voluntária dos questionários online. O protocolo do es- tudo foi aprovado pela comissão de ética da ARS Norte.

\section{RESULTADOS}

Dos 1.976 indivíduos para os quais foi enviado o formulário online obtiveram-se 450 respostas $(22,8 \%)$, tendo sido incluídos no estudo 359 médicos de família ( $\mathrm{Fi}$ gura 1).

A mediana da idade dos especialistas foi de 56 anos com amplitude interquartil de 22. As idades mínima e máxima dos especialistas no estudo foram 30 e 65 anos, respetivamente. Relativamente aos anos de atividade como MF, a mediana foi de 20 anos com amplitude interquartil de 23. Quanto ao número de colegas médicos na mesma unidade, a mediana foi de seis com amplitude interquartil de dois. No Quadro I são apresentadas as características dos participantes relativas às variáveis qualitativas.

A EE foi a dimensão mais afetada, apresentando uma maior prevalência para níveis altos, contrariamente à DP, que apresentou uma menor taxa de especialistas com níveis altos (Quadro II).

A prevalência bruta da síndroma de Burnout [exaustão emocional (EE) e despersonalização (DP) altas e realização profissional (RP) baixa] foi de 17,0\% (IC95\%: $13,1-20,9)$. 


\begin{tabular}{|c|c|c|c|c|c|c|}
\hline & $\begin{array}{c}\text { Exaustão } \\
\text { emocional }\end{array}$ & \% (IC95\%) & Despersonalização & $\%$ (IC95\%) & $\begin{array}{l}\text { Realização } \\
\text { profissional }\end{array}$ & \% (IC95\%) \\
\hline Alta & 237 & $66,0(61,1-70,9)$ & 164 & $45,7(40,5-50,9)$ & 173 & $28,4(23,7-33,1)$ \\
\hline Intermédia & 81 & $22,6(18,2-26,9)$ & 66 & $18,4(14,4-22,4)$ & 84 & $23,4(19,0-27,8)$ \\
\hline Baixa & 41 & $11,4(8,1-14,7)$ & 129 & $35,9(30,9-40,9)$ & 102 & $48,2(43,0-53,4)$ \\
\hline
\end{tabular}

A Figura 2 representa os indivíduos afetados por EE alta, DP alta e/ou RP baixa isoladamente ou em combinação.

No presente estudo constatou-se que a despersonalização alta foi significativamente mais frequente nos indivíduos com menos de 45 anos $(p=0,016)$ e nos indivíduos do sexo masculino $(p=0,034)$. A prevalência da síndroma de Burnout foi significativamente maior nos indivíduos com menos de 45 anos de idade $(p=0,028)$ e nos indivíduos com menos de 20 anos de atividade como MF ( $p=0,018)$. Não foram encontradas associações estatisticamente significativas entre a síndroma e a carga horária contratual semanal, o número médio de horas diárias de contacto com utentes ou o número de utentes (Quadro III).

\section{DISCUSSÃO}

No presente estudo constatou-se que a prevalência da síndroma de Burnout foi quatro vezes maior que o valor obtido num estudo português de âmbito nacional publicado em 2012, segundo a mesma definição para a síndroma de Burnout (EE e DP altas e RP baixa). Em relação a cada uma das dimensões, comparativamente ao estudo publicado em 2012, a EE alta, a DP alta e a RP baixa foram, respetivamente, 2,6, 2,8 e 2,9 vezes maiores. ${ }^{9}$ Um outro estudo publicado em 2016, realizado apenas num Agrupamento de Centros de Saúde (na região centro de Portugal), apresentou uma prevalência de síndroma de Burnout também inferior ao presente estudo. ${ }^{17}$ Comparativamente aos valores encontrados num estudo multicêntrico em vários países europeus (em que Portugal não participou), o presente estudo apresentou uma prevalência superior; contudo, a diferença foi menos discrepante. ${ }^{10} \mathrm{~A}$ comparação dos dados obtidos com os outros estudos encontram-se descritos na Quadro IV.

Em relação a fatores associados à síndroma de Burnout e suas dimensões verificou-se que os especialistas do sexo masculino têm níveis de despersonalização significativamente mais altos do que as mulheres, à semelhança do estudo europeu e do estudo português publicado em 2012. ${ }^{9-10}$ De acordo com Maslach, os homens são mais afetados na dimensão da despersonalização por apresentarem atitude mais cínica face ao stress, ou seja, manifestam indiferença e desvalorização sobre os problemas encontrados no dia-a-dia da sua profissão, perpetuando o
Figura 2. Diagrama de Venn da distribuição dos participantes pela exaustão emocional (EE) alta, despersonalização (DP) alta e/ou realização profissional (RP) baixa, $N=359$. 
meio nocivo e conduzindo à síndroma. ${ }^{5}$

A faixa etária com menos de 45 anos apresentou significativamente maior prevalência de despersonalização alta e síndroma de Burnout, à semelhança do estudo português publicado em 2016 mas em oposição ao estudo português publicado em 2012, que não demonstrou ser significativamente diferente. ${ }^{9,17}$ No estudo europeu também foi descrita uma maior prevalência da síndroma nas faixas etárias mais novas. ${ }^{10} \mathrm{~A}$ associação entre os indivíduos mais jovens com a síndroma é controversa entre os diversos autores; contudo, segundo uma meta-análise realizada em 2004, a anterior associação é significativa apesar de pequena e poderá estar relacionada com o facto de que indivíduos mais jovens têm uma menor capacidade de lidar com o stress e, consequentemente, de serem mais suscetíveis à síndroma. ${ }^{18}$

Também foi encontrada uma associação significativa entre os anos de atividade como MF inferior a 20 com a síndroma de Burnout. Tal achado poderá resultar de os indivíduos mais novos terem geralmente menos anos de atividade.

Apesar de um número absoluto de respostas satisfatório, a relativamente baixa proporção de respostas obtida não permite transpor a prevalência da síndroma de Burnout e respetivas associações encontradas a todos os especialistas de MGF da SRNOM. A comparação entre especialistas respondentes e não respondentes seria útil, mas não foi possível por não se disporem de dados sobre os não respondentes nem sobre a população total (especialistas de MGF da SRNOM).

Algo que deverá ser considerado é o método de recrutamento por via online, ou seja, um método mais impessoal que contribuiu provavelmente para a baixa proporção de respostas, à semelhança de estudos com os mesmos moldes. ${ }^{10}$ Contudo, no presente estudo a proporção de respostas foi inferior, comparativamente a estudos similares (Quadro IV).9-10,17

Perante esta situação, é necessário ter em conta que os especialistas que responderam podem ter uma prevalência da síndroma de Burnout diferente da que teriam os que não responderam.

Por um lado, poderá ser possível que especialistas com síndroma de Burnout tenham uma maior propensão para responder a um questionário sobre o mesmo tema, sobrestimando a prevalência. Os indivíduos com síndroma de Burnoutapresentam mais autoconsciência, assim como os mais suscetíveis à síndroma são mais do «tipo emocional» do que do «tipo reflexivo», ou seja, o indivíduo passaria a assumir comportamentos mais efetivos na resolução dos problemas, por exemplo, a procura de ajuda, refletida na resposta ao questionário. ${ }^{5}$

Por outro lado, é também possível que especialistas sem síndroma de Burnout possam ter mais tempo disponível para responder ao questionário, subestimando a prevalência. De acordo com Maslach, a síndroma de Burnout associa-se a comportamentos do tipo A, onde se inclui o estilo de vida com pressão de tempo e cinismo com atitude de indiferença para a própria exaustão que, por sua vez, poderá conduzir a uma menor predisposição à resposta do questionário. ${ }^{5}$

O método de recrutamento por via online está associado a uma maior resposta ao questionário por parte de indivíduos mais jovens e do sexo masculino. ${ }^{19}$ Consequentemente, uma vez que a síndroma de Burnouté mais prevalente nos grupos mais jovens, a prevalência calculada neste estudo poderá ser maior do que a real prevalência da síndroma nos especialistas de MGF.18

No método utilizado não foi possível impedir que os especialistas, que já teriam respondido ao questionário, respondessem novamente; existe, portanto, a possibilidade de duplicados. Para minimizar esta possibilidade, o envio dos lembretes foi realizado em intervalos de tempo curtos.

Alguns dos especialistas não se encontravam na lista da SRNOM. Porém, a própria SRNOM estimou que o número destas situações fosse mínimo, pelo que se pressupõe que o impacto sobre o estudo seja nulo ou desprezível.

É de considerar ainda a hipótese dos especialistas de MGF participantes, maioritariamente do norte do país, serem diferentes demograficamente ou em outros aspetos em relação aos especialistas do centro, sul do país e regiões autónomas e que, por isso, dificulte a transposição dos resultados do presente estudo para todos os especialistas de MGF portugueses.

Os achados do presente estudo poderão ser extrapolados para os restantes especialistas de MGF portugueses de forma a permitir uma reflexão sobre a tendência do aumento da prevalência da síndroma de Burnout encontrada em Portugal e na Europa, até pelo fac- 


\begin{tabular}{|c|c|c|c|c|c|c|c|c|c|c|c|c|c|}
\hline \multirow{4}{*}{\multicolumn{2}{|c|}{ 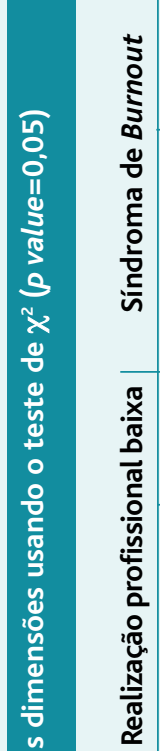 }} & \multicolumn{2}{|c|}{ 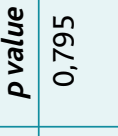 } & : & \multicolumn{2}{|l|}{$\begin{array}{l}\tilde{y} \\
\tilde{m} \\
\tilde{c} \\
0\end{array}$} & 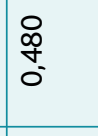 & $\frac{\infty}{0}$ & $\begin{array}{l}\text { Ln } \\
\infty \\
\infty \\
0\end{array}$ & $\begin{array}{l}\text { On } \\
\text { مू } \\
0\end{array}$ & \begin{tabular}{l}
$\infty$ \\
\multirow{2}{n}{} \\
0 \\
0 \\
0
\end{tabular} & $\begin{array}{l}\overline{0} \\
\stackrel{\text { L }}{0}\end{array}$ & $\begin{array}{l}\stackrel{L}{\infty} \\
\stackrel{0}{0} \\
0\end{array}$ \\
\hline & & 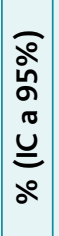 & 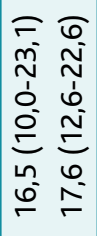 & 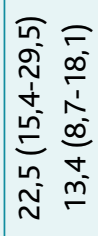 & 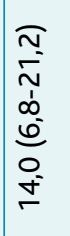 & 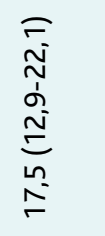 & 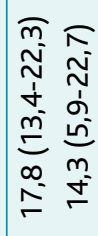 & 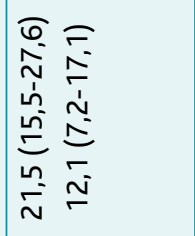 & 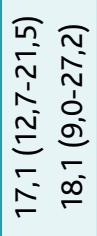 & 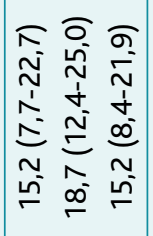 & 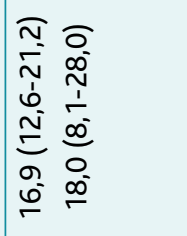 & 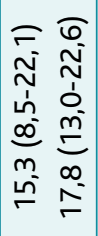 & 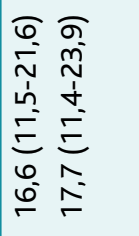 \\
\hline & & $\begin{array}{l}\frac{1}{3} \\
\frac{\pi}{2} \\
a\end{array}$ & 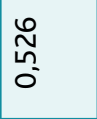 & 恣 & $\begin{array}{l}0 \\
6 \\
0 \\
0\end{array}$ & & 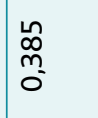 & 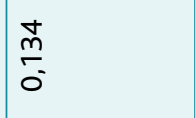 & $\frac{0}{m}$ & 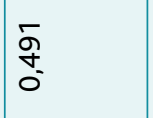 & 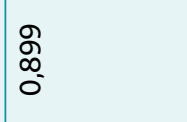 & 离 & $\frac{5}{6}$ \\
\hline & & 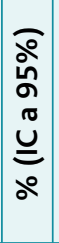 & 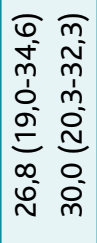 & 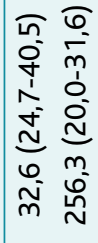 & $\begin{array}{l}0 \\
0 \\
0 \\
m \\
\dot{0} \\
0 \\
0 \\
\vdots \\
\overline{0} \\
m\end{array}$ & 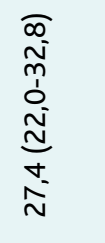 & 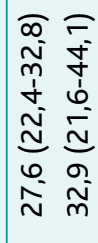 & 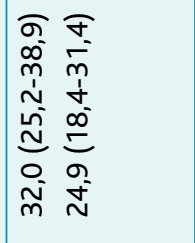 & 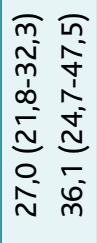 & 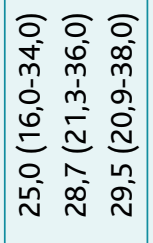 & 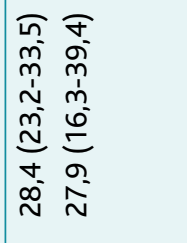 & 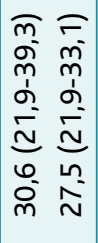 & 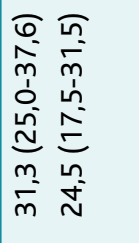 \\
\hline 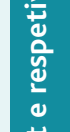 & $\begin{array}{l}\frac{\pi}{ \pm} \\
\frac{\pi}{0} \\
\stackrel{0}{2} \\
2\end{array}$ & $\begin{array}{c}\frac{2}{3} \\
\frac{\pi}{3} \\
a \\
\end{array}$ & $\begin{array}{l}\stackrel{1}{m} \\
0 \\
0\end{array}$ & 음 & 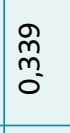 & & $\begin{array}{l}a \\
\hat{R} \\
0\end{array}$ & 兽 & $\begin{array}{l}8 \\
\infty \\
0 \\
0\end{array}$ & $\begin{array}{c}\text { ñ } \\
\text { m. } \\
0\end{array}$ & $\begin{array}{l}0 \\
\infty \\
0 \\
0 \\
0\end{array}$ & \begin{tabular}{l}
\multirow{2}{0}{} \\
O \\
-
\end{tabular} & $\begin{array}{c}N \\
N \\
0 \\
0\end{array}$ \\
\hline 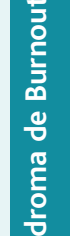 & 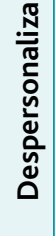 & 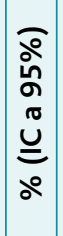 & 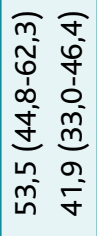 & 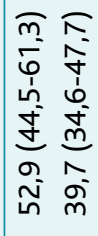 & 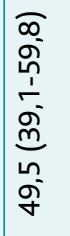 & 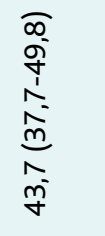 & 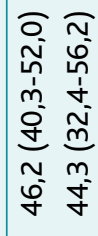 & 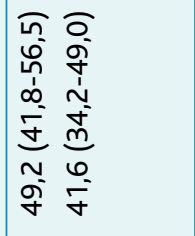 & 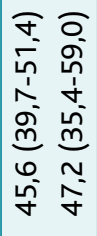 & 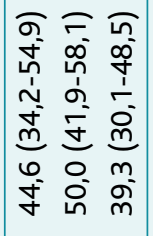 & 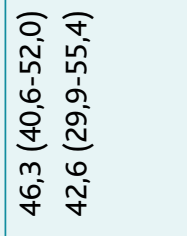 & 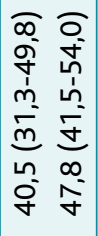 & 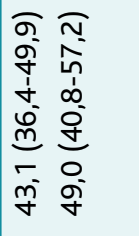 \\
\hline s & $\begin{array}{l}\frac{\pi}{\pi} \\
\frac{\pi}{\pi} \\
\frac{\pi}{0}\end{array}$ & $\begin{array}{l}\frac{9}{3} \\
\frac{\pi}{2} \\
0\end{array}$ & 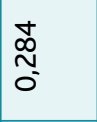 & 䒹 & $\frac{0}{\tilde{N}}$ & & 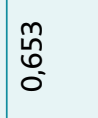 & 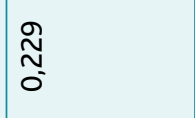 & $\begin{array}{l}8 \\
0 \\
0 \\
0\end{array}$ & 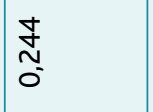 & $\begin{array}{c}\bar{\sigma} \\
0 \\
0\end{array}$ & $\begin{array}{l}J \\
⿱ \\
0\end{array}$ & $\begin{array}{l}\hat{n} \\
\infty \\
0 \\
0\end{array}$ \\
\hline$\frac{d}{c}$ & 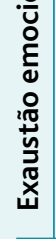 & 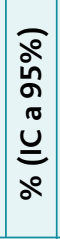 & 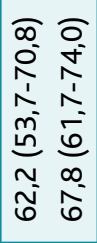 & $\begin{array}{ll}0 & \pi \\
0 & 0 \\
0 & 0 \\
0 & 1 \\
i & 0 \\
0 & 0 \\
0 & 0 \\
0 & 0 \\
0 & 0 \\
0 & 0 \\
0 & 0\end{array}$ & $\begin{array}{l}0 \\
0 \\
0 \\
0 \\
0 \\
0 \\
0 \\
0 \\
0 \\
0 \\
i\end{array}$ & 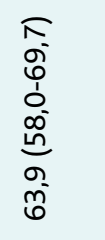 & 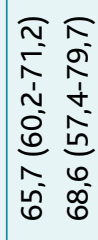 & 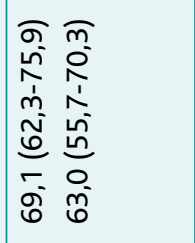 & 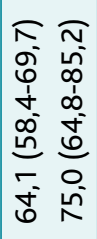 & 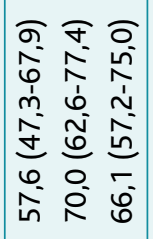 & 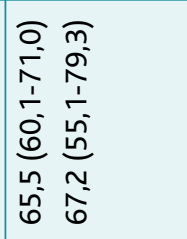 & 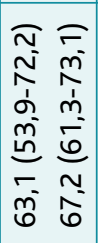 & 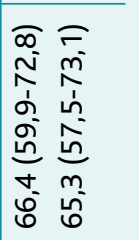 \\
\hline 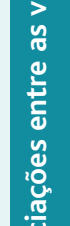 & & & 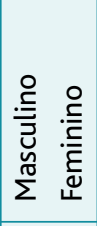 & 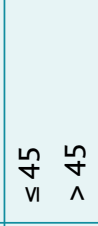 & 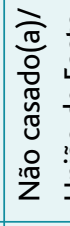 & 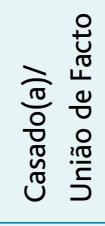 & 结 $\frac{20}{2}$ & 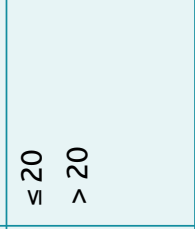 & 岕 & 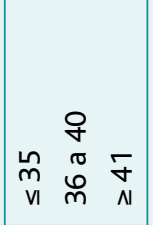 & $\begin{array}{ll}a & \sigma \\
v & \Lambda\end{array}$ & 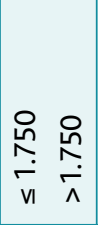 & $\begin{array}{ll}0 & 0 \\
\mathrm{VI} & 1\end{array}$ \\
\hline $\begin{array}{l}8 \\
\\
c \\
c \\
c\end{array}$ & 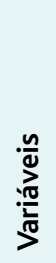 & & $\stackrel{\substack{x \\
凶}}{n}$ & 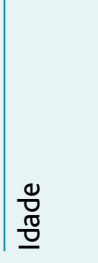 & 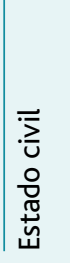 & & 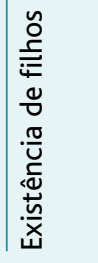 & 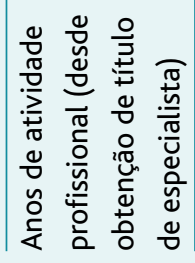 & 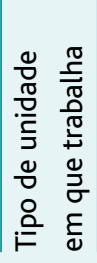 & 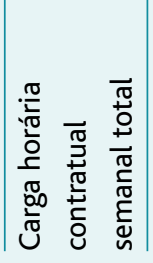 & 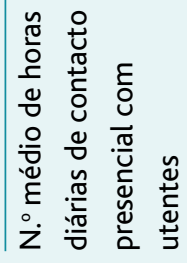 & 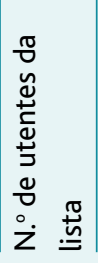 & 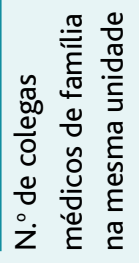 \\
\hline
\end{tabular}


QUADRO IV. Comparação da prevalência da síndroma de Burnout nos médicos de família e suas dimensões entre estudos similares em Portugal e Europa

\begin{tabular}{|l|c|c|c|c|c|c|c|} 
& $\begin{array}{c}\text { Local de } \\
\text { estudo }\end{array}$ & $\begin{array}{c}\% \\
\text { respostas }\end{array}$ & $\boldsymbol{n}$ válido & $\begin{array}{c}\text { \% EE } \\
\text { alta }\end{array}$ & $\begin{array}{c}\text { \% DP } \\
\text { alta }\end{array}$ & $\begin{array}{c}\text { \% RP } \\
\text { baixa }\end{array}$ & $\begin{array}{c}\text { \% Síndroma } \\
\text { de Burnout }\end{array}$ \\
\hline Soler, et al. (2008) ${ }^{10}$ & Europa & 40,8 & 1.393 & 43,0 & 35,3 & 39,5 & 12,0 \\
\hline Marcelino, et al. (2012) & Portugal & 41,2 & 150 & 25,3 & 16,2 & 16,7 & 4,1 \\
\hline Mata, et al. (2016) & Portugal & 60,1 & 83 & 47,0 & 33,7 & 21,7 & 7,2 \\
\hline Presente estudo & Portugal & 22,7 & 359 & 66,0 & 45,7 & 48,2 & 17,0 \\
\hline
\end{tabular}

Legenda: $\mathrm{EE}=$ exaustão profissional; $\mathrm{DP}=$ despersonalização; RP = realização profissional. idades inferiores a 45 anos. A síndroma de Burnout também esteve associada a idade inferior a 45 anos e a uma duração da atividade como MF inferior a 20 anos.

\section{AGRADECIMENTOS}

Agradece-se à Dra. Mónica Granja, pela sua contribui-

to de terem sido encontradas as mesmas associações a determinados fatores de outros estudos em $\mathrm{MF}^{9-10,17} \mathrm{O}$ presente estudo foi o maior realizado em especialistas de MGF portugueses até ao momento, contando com uma grande amostra, tendo sido adoptado o mesmo questionário (MBI-HSS), validado para português europeu e aplicado em estudos similares, facilitando a comparação com os mesmos.

Dada a forma de recrutamento dos participantes foi prevista uma taxa de resposta baixa e para a minimizar enviaram-se dois lembretes de forma a maximizar as respostas. Contudo, a proporção de respostas foi inferior comparativamente a outros estudos. Deverão ser exploradas estratégias que possam maximizar o número de respostas, por exemplo, o incentivo e a comunicação de entidades competentes dos estudos em curso ou o estabelecimento de um grupo de estudo que invista em métodos de recrutamento mais pessoais.

Por se tratar de um estudo transversal, não foi possível aferir a relação causal dos fatores com o aumento relatado. Nota-se, portanto, a necessidade de um estudo longitudinal para averiguar e quantificar o impacto dos anteriores fatores.

\section{CONCLUSÕES}

A prevalência da síndroma de Burnout foi de 17,0\%, quatro vezes maior quando comparada com o estudo português publicado em 2012; de igual modo superior ao estudo português de 2016 e ao estudo europeu de 2008.

As associações encontradas são consistentes às verificadas em estudos anteriores. A despersonalização associou-se ao sexo masculino e aos indivíduos com ção na revisão e análise crítica do protocolo e da redação do artigo, e ao Dr. Hugo Rodrigues, representante eleito pela Secção Regional Norte da Ordem dos Médicos, pelo sistema de envio dos questionários via online e respetivo envio, assim como à própria Secção Regional Norte da Ordem dos Médicos por permitir o envio dos questionários.

\section{REFERÊNCIAS BIBLIOGRÁFICAS}

1. Allen J, Gay B, Crebolder H, Heyrman J, Svab I, Ram P, et al. The European definition of General Practice / Family Medicine [Internet]. 3rd ed. Brussels: WONCA Europe; 2015. Available from: http://www.woncaeurope.org/sites/default/files/documents/Definition\%203rd\%20ed \%202011\%20with\%20revised\%20wonca\%20tree.pdf

2. Schultz K, Delva D, Kerr J. Emotional effects of continuity of care on family physicians and the therapeutic relationship. Can Fam Physician. 2012;58(2):178-85

3. Woolhouse S, Brown JB, Thind A. Building through the trauma in a group of inner-city family physicians. J Am Board Fam Med. 2012;25(6): 840-6.

4. Romani M, Ashkar K. Burnout among physicians. Libyan J Med. 2014;9: 23556.

5. Maslach C, Schaufeli WB, Leiter MP. Job burnout. Annu Rev Psychol. 2001;52:397-422.

6. Maslach C, Jackson SE, Leiter MP, Schaufeli WB. The Maslach Burnout Inventory. 3rd ed. Palo Alto: Consulting Psychologists Press; 1996. ISBN 9789996345777

7. Marcelino G, Cerveira JM, Carvalho I, Costa JA, Lopes M, Calado NE, et al. MBI-HSS validity in portuguese medical doctors. In: Doolittle BR, editor. Psychology of Burnout: new research. New York: Nova Science Publishers; 2013. p. 121-35. ISBN 9781629483139

8. Carlotto MS, Câmara SG. Propriedades psicométricas do Maslach Burnout Inventory em uma amostra multifuncional [Psychometrics properties of Maslach Burnout Inventory in a multifunctional sample. Estud Psicol. 2007;24(3):325-32. Portuguese

9. Marcelino G, Cerveira JM, Carvalho I, Costa JA, Lopes M, Calado NE, et al. Burnout levels among Portuguese family doctors: a nationwide survey. BMJ Open. 2012;2:e001050.

10. Soler JK, Yaman H, Esteva M, Dobbs F, Asenova RS, Katic M, et al. Burnout in European family doctors: the EGPRN study. Fam Pract. 2008;25(4):245-65. 
11. Perelman J, Felix S, Santana R. The great recession in Portugal: impact on hospital care use. Health Policy. 2015;119(3):307-15.

12. Costa FA, Pedro AR, Teixeira I, Bragança F, da Silva JA, Cabrita J. Primary non-adherence in Portugal: findings and implications. Int J Clin Pharm. 2015;37(4):626-35.

13. Karanikolos M, Mladovsky P, Cylus J, Thomson S, Basu S, Stuckler D, et al. Financial crisis, austerity, and health in Europe. Lancet. 2013;381 (9874):1323-31.

14. Zivin K, Paczkowski M, Galea S. Economic downturns and population mental health: research findings, gaps, challenges and priorities. Psychol Med. 2011;41(7):1343-8.

15. Santana P, Peixoto H, Duarte N. Demography of physicians in Portugal: prospective analysis [Demography of physicians in Portugal: prospective analysis]. Acta Med Port. 2014;27(2):246-51. Portuguese

16. Decreto-Lei n. ${ }^{\circ}$ 266-D/2012, de 31 de dezembro. Diário da República. 1. ${ }^{a}$ Série(252).

17. Mata C, Machado S, Moutinho A, Alexandra D. Estudo PreSBurn: prevalência de síndroma de Burnout nos profissionais dos cuidados de saúde primários [PreSBurn study: prevalence of Burnout syndrome in pri- mary care professionals]. Rev Port Med Geral Fam. 2016;32(3):179-86. Portuguese

18. Brewer EW, Shapard L. Employee Burnout: a meta-analysis of the relationship between age or years of experience. Hum Resour Dev Rev. 2016;3(2):102-23.

19. Schmidt WC. World-wide web survey research: benefits, potential problems, and solutions. Behav Res Methods Instrum Comput. 1997;29(2): 274-9.

\section{CONFLITO DE INTERESSES}

$\mathrm{O}$ autor declara não ter quaisquer conflitos de interesse.

\section{ENDEREÇO PARA CORRESPONDÊNCIA}

Carlos Daniel Costa Reis

E-mail: carlosreis.sketch@gmail.com

http://orcid.org/0000-0001-6739-0291

Recebido em 10-07-2017

Aceite para publicação em 11-01-2019

\section{ABSTRACT}

\section{PREVALENCE OF BURNOUT SYNDROME IN FAMILY DOCTORS OF THE NORTH REGIONAL SECTION OF THE PHYSICIANS' ORDER}

Objectives: Family physicians are health professionals who make the first contact between patients and the health system, having a continuous and close interaction with the patients, which can trigger anxiety and fear. Negative feelings enhance physical and mental exhaustion that could lead to Burnout. The recent Portuguese conjuncture presents factors that could increase the prevalence of Burnout syndrome in family physicians. This study aims to re-evaluate the prevalence of Burnout syndrome in family physicians of the Northern Regional Section of the Medical Association.

Design: Cross-sectional, observational and descriptive study with an analytical component.

Local: Portugal.

Population: Physicians enrolled as family physicians in the Northern Regional Section of the Medical Association.

Methods: This study was conducted through an online questionnaire consisting of demographic and professional data and the Maslach Burnout Inventory-Human Services Survey, validated in Portuguese, sent to family physicians in the form of a census on 11 November 2016 and valid for submission of answers until 5 December 2016.

Results: The prevalence of Burnout found in family physicians was $17.0 \%(95 \% \mathrm{Cl} ; 13.1-20.9)$. The family physicians presented $66.0 \%$ with high emotional exhaustion $(95 \% \mathrm{Cl} ; 61.1-70.9), 45.7 \%$ with high depersonalization $(95 \% \mathrm{Cl} ; 40.5-50.9)$ and $48.2 \%$ with low professional achievement $(95 \% \mathrm{Cl} ; 43.0-53.4)$. Depersonalization was associated with males and individuals younger than 45 years. Burnout syndrome was also associated with age less than 45 years and with a duration of family physician activity of fewer than 20 years.

Conclusions: The prevalence of Burnout syndrome was up to four times higher than the values obtained in previous studies. The associations found are consistent with those found in previous studies, and the syndrome is more prevalent in younger doctors with less experience as a family doctor.

Keywords: Prevalence; Stress, Psychological; Burnout, Professional; Physicians, Family; Portugal. 